\title{
Die Medizin wird besser - Matisse bleibt gut
}

\section{Felix Schürch}

Dr. med., Facharzt für Allgemeine Innere Medizin, Mitglied FMH

Mit dem Begriff «Metamorphosen» bezeichnen wir Umwandlungen in der Pflanzen- und in der Tierwelt, aber auch die Verwandlung von Fabelwesen in den mythologischen Erzählungen. Die Ausstellung «Matisse - Metamorphosen» in Zürich stellt uns den Maler und Bildhauer Henri Matisse vor. Sie zeigt Werke des Künstlers in verschiedenen Versionen und Stadien der Bearbeitung. Die Ausstellung regt zur Reflexion darüber an, was Entwicklung bedeutet: In der Kunst - und in der Medizin. So betrachtet ist es eine Ausstellung zum Hinsehen und zum Nachdenken.

Die Überschrift zu dieser Ausstellungsbesprechung ist etwas salopp gewählt - der Titel variiert einen Werbespruch für ein Mineralwasser aus den Bündner Bergen [1]. Dabei geht es hier um Henri Matisse, einen der ganz Grossen der modernen Malerei - und wie in Zürich gezeigt wird: auch der Bildhauerkunst. Aber der Titel umschreibt, was mir nach dem Rundgang durch die Ausstellung bei einem Glas Rosé im Restaurant des Museums durch den Kopf ging: Gedanken zu Fortschritt, Kunst und Medizin.

Doch zunächst zur Ausstellung. Im Mittelpunkt stehen vier Reliefs aus Bronze, jedes knapp einen Meter breit und gut zwei Meter hoch. Sie alle zeigen eine weibliche Figur als Rückenakt. Die erste Version - «Nu de dos I»stammt aus dem Jahre 1908, die vierte Version wurde 1930 geschaffen. Bei dieser letzten Ausführung sind die Konturen des weiblichen Körpers auf geometrische Formen reduziert. Eine Fotografie aus dem Jahre 1953 zeigt uns das Werk als Gipsskulptur neben der Türe des Ateliers deponiert. Neun Museen auf der Welt, darunter das Kunsthaus Zürich, besitzen in ihrer Sammlung von den Rückenakten das ganze Set I bis IV in Bronze [2]. Diese Reliefs sind im bildhauerischen Werk von Matisse in Bezug auf das Format aussergewöhnlich, die Skulpturen des Künstlers sind im Allgemeinen klein gehalten. Aber wie bei den grossen Reliefs werden wir auch da Augenzeugen eines schöpferischen Prozesses, sei das bei den verschiedenen Versionen eines Torsos, einer Figur oder einer Büste.

Sind diese Metamorphosen im Atelier des Künstlers ein zielloses Spiel? Oder gibt es Fortschritte, einen Weg

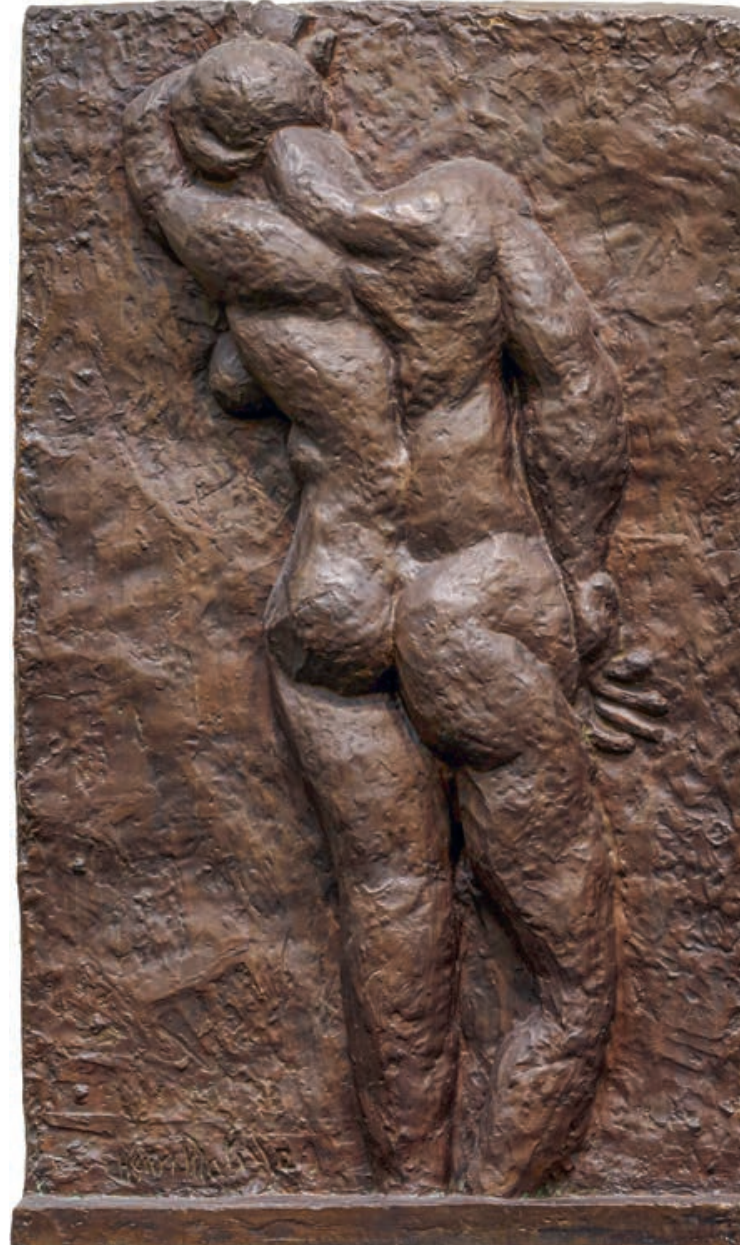

Henri Matisse, Nu de dos I, 1908-1909.

Bronze, $190 \times 116 \times 15$ cm, Kunsthaus Zürich, 1960

(๔ Succession Henri Matisse / 2019 ProLitteris, Zurich). 


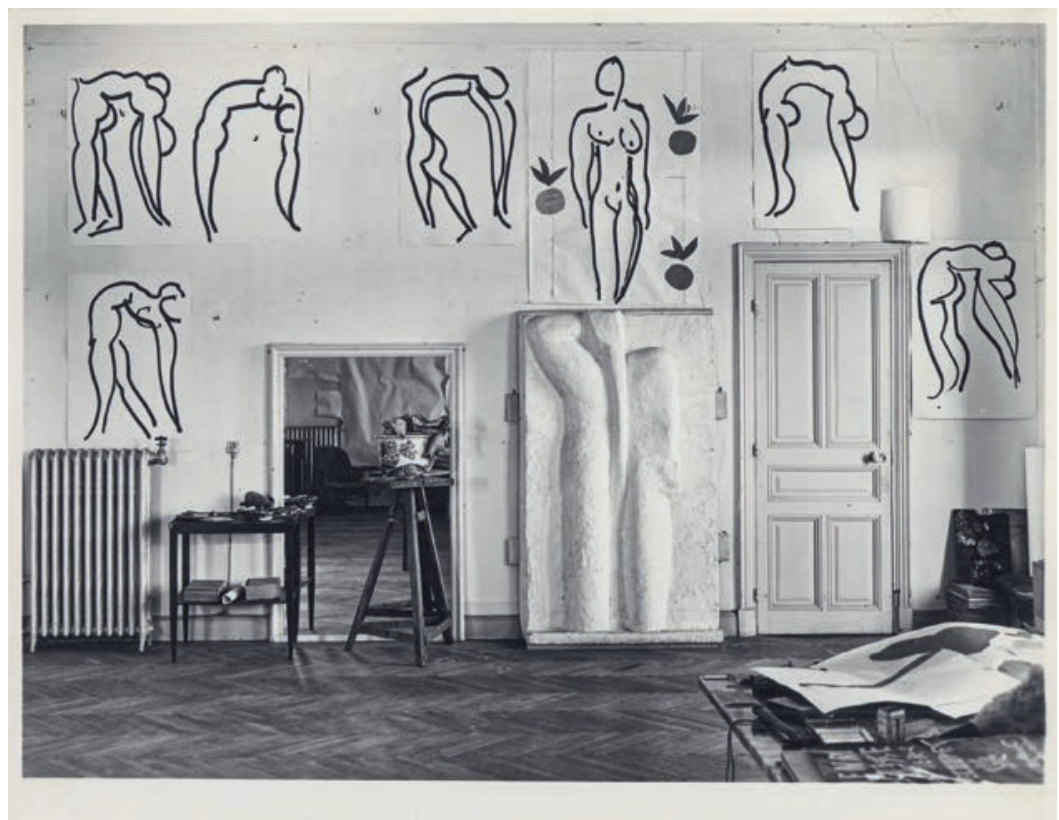

Atelier von Henri Matisse im ehemaligen Hôtel Regina in Cimiez bei Nizza, 1953. Archives Henri Matisse, Issy-les-Moulineaux (@ Succession Henri Matisse / 2019 ProLitteris, Zurich).
Dr. med. Felix Schürch Albulastrasse 52

CH-8048 Züric

felix.schurch[at]hin.ch zum Besseren, von Meilenstein zu Meilenstein? - Wohl kaum. Wer könnte uns besser darüber aufklären als die Künstler selber. Matisse hatte grossen Respekt vor der Kunst der Antike, vor den Skulpturen von Auguste Rodin oder der "primitiven" Kunst Afrikas. Andy Warhol, der Meister der Pop-Art, drückte seine Bewunderung der Werke von Henri Matisse mit dem Ausspruch aus: «Ich möchte Matisse sein.» Pablo Picasso besuchte 1940 die steinzeitlichen Felsenmalereien in Südfrankreich. Beim Anblick der jahrtausendealten Zeichnungen soll er ausgerufen haben: «Wir haben nichts dazugelernt.»

Wie steht es da mit der Heilkunst? Um die Lernprozesse in der Medizin? Hier gibt es zweifellos Fortschritte! Auf jeden Fall bei den naturwissenschaftlichen Erkenntnissen zu den Krankheitsvorgängen und bei den modernen Möglichkeiten in der Diagnostik und in der Therapie. Gerade der Lebenslauf von Henri Matisse (1869-1954) gibt uns ausgiebig Anschauungsunterricht. Ernst Gemsenjäger, ehemaliger Chefarzt der Viszeralchirurgie, verfasste 2017 eine Fallbesprechung «über die Krankheiten und Operationen von Henri Matisse» [3]. Darin wird ein monatelang andauerndes Beschwerdebild mit Schmerzattacken und abdominellen Krämpfen geschildert. Man diagnostiziert Gallensteine, im Röntgen stellt man eine Stenose im
Dickdarm fest. 1941 erfolgt beim 71-jährigen Patienten eine Operation, bei der das Sigma mitsamt einem gutartigen Tumor entfernt wird. Es ist leicht vorstellbar, dass die Voruntersuchungen heute mit der zur Verfügung stehenden Bildgebung und der Endoskopie rascher und präziser zur Diagnose führen würden. Die Dickdarmoperation könnte auf weniger invasive Art ausgeführt werden, und der Verlauf wäre wohl mit weniger Komplikationen behaftet. Gemsenjäger sagt es so: «Die medizinische Biographie Matisse' macht deutlich, welch echte, grosse Fortschritte Medizin und Chirurgie in wenigen Dezennien erfahren haben, etwa bei der Erkennung und Therapie der 'Syndromes douloureux de la région épigastrique', des Steinbefalls der Gallenwege, bei der Bewerkstelligung einer Kolonsegment-Resektion mit einer primär heilenden Stoss-aufStoss-Darmnaht mit modernem atraumatischen Nahtmaterial» ([3], S. 26).

Der Patient Henri Matisse konnte von diesen Fortschritten der Medizin nicht profitieren. Nach der Operation vom 16. Januar 1941 kommt es zu belastenden Komplikationen: Wundinfekt, Lungenembolie, Platzbauch, ein künstlicher Darmausgang, der nicht zurückverlegt werden kann. Und in den folgenden Jahren macht sich das Gallensteinleiden wiederholt mit quälenden und lebensbedrohlichen Attacken bemerkbar. Die behandelnden Ärzte - Hausarzt, Radiologe, Gastroenterologe und Chirurg - stehen offenbar in einem regen fachlichen Austausch. Der Patient wird von den Ärzten gründlich aufgeklärt, davon zeugen die Skizzen zur Kolonresektion, die Matisse in seine private Korrespondenz einfügt ([3], S. 73). Der informierte Patient Matisse wird sich später ganz bewusst gegen eine Gallenblasenoperation entscheiden. Bei diesen Aspekten in der Krankengeschichte des Künstlers sehe ich moderne Züge - vielleicht passt sogar der Begriff «zeitlos».

\section{Literatur}

1 In den 1990er Jahren erfanden die Werber von Contexta den Slogan für das Valser Mineralwasser: "Alles wird besser - Valser bleibt gut.»

2 Wikipedia [Internet]: https://en.wikipedia.org/wiki/The_Back_ Series

3 Gemsenjäger E. Die Krankheiten und Operationen von Henri Matisse. Basel: EMH Schweizerischer Ärzteverlag; 2017.

Ausstellung, bis 8. Dezember 2019

Matisse - Matamorphosen

Meilenstein in der Skulptur der Moderne

Kunsthaus Zürich 